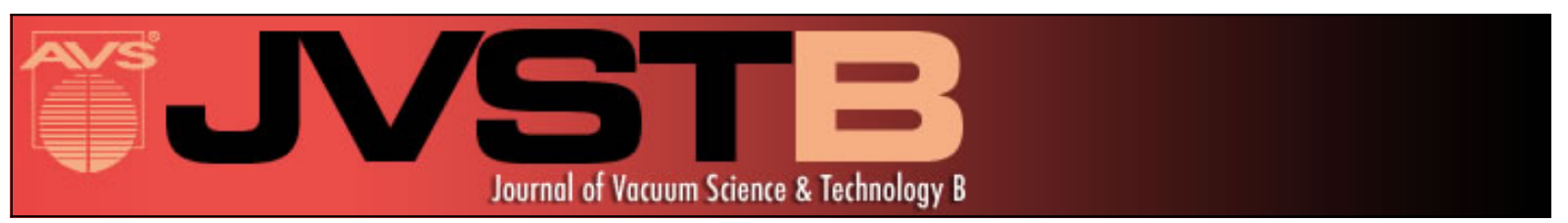

Evolution of Physics and Chemistry of Surfaces and Interfaces: A Perspective of the

\title{
Last 40 Years
}

David K. Ferry

Citation: Journal of Vacuum Science \& Technology B 31, 048501 (2013); doi: 10.1116/1.4806761

Vublished by the AVS: Science \& Technology of Materials, Interfaces,

View Table of Contents: http://scitation.aip.org/content/avs/journal/jvstb/31/4?ver=pdfcov

View Table of Contents: http://scitation.aip.org/content/avs/journal/jvstb/31/4?ver=pdfcov

\section{Instruments for advanced science}
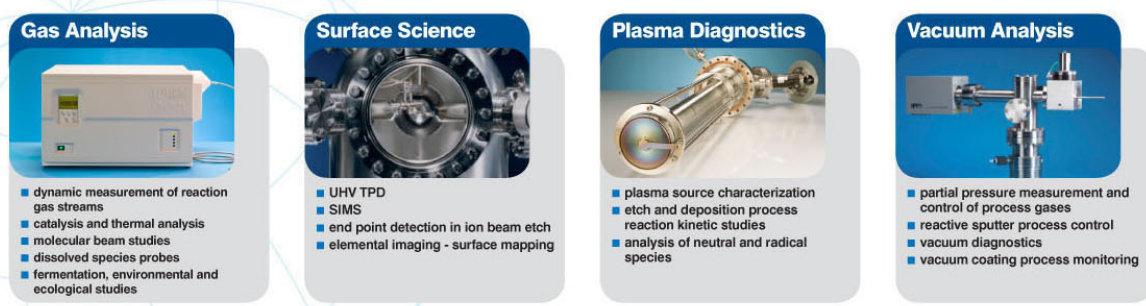

contact Hiden Analytical for further details

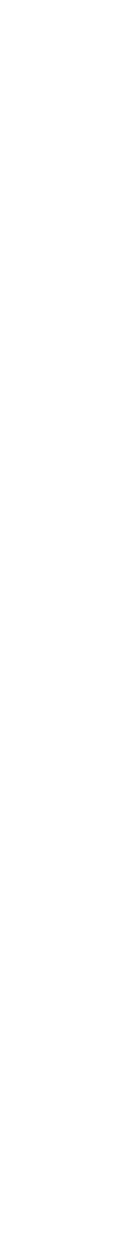




\title{
Evolution of Physics and Chemistry of Surfaces and Interfaces: A Perspective of the Last 40 Years
}

\author{
David K. Ferry ${ }^{\text {a) }}$ \\ School of Electrical, Computer, and Energy Engineering, Arizona State University, Tempe, Arizona 85287
}

(Received 25 March 2013; accepted 29 April 2013; published 17 May 2013)

\begin{abstract}
The Physics and Chemistry of Surfaces and Interfaces conference has maintained a focus on the interfacial and surface properties of materials since its initiation in 1974. The conference continues to be a major force in this field, bringing together scientists from a variety of disciplines to focus upon the science of interfaces and surfaces. Here, a historical view of the development of the conference and a discussion of some of the themes that have been focal points for many years are presented. (C) 2013 American Vacuum Society. [http://dx.doi.org/10.1116/1.4806761]
\end{abstract}

\section{INTRODUCTION}

In the early Fall of 1973, Horst Wittman and I were discussing the lack of a forum for addressing the scientific issues concerning the physics of surfaces and interfaces in the III-V compounds. As has been rumored, these discussions took place in a bar at the Los Angeles airport while awaiting flights back to the east coast. At the time, Horst was with the Army Research Office, while I was with the Office of Naval Research. So, we both had considerable exposure to researchers working in this important area; at least the area was important to our programs on future electronics. The result of these discussions was a plan to create a workshop which would bring together a number of the leading people in the field to discuss germane questions, providing of course that we could draw them to the meeting.

Upon returning to the east coast, the concept was discussed with Larry Cooper, also with the Office of Naval Research, and the consensus was to give the workshop a try. The first workshop would be organized by Carl Wilmsen and Jim Sites, and held at Colorado State University in January 1974. The resulting success of this first workshop led to a continuation of the idea on an annual basis. This has become the series of Physics and Chemistry of Surfaces and Interfaces (PCSI) workshops.

The name has evolved over the years, as the scope of the workshop has evolved. The first series of workshops were titled Physics of Compound Semiconductor Interfaces, and the sites of this first series are shown in Table I. Following the 1981 workshop, it was decided to broaden the scope to incorporate studies, which could be classified as more chemistry related. This led to a change in the title to Physics and Chemistry of Semiconductor Interfaces. The sites for this second series of workshops are shown in Table II. Finally, in 2008 , the scope was broadened once again to expand beyond semiconductors and include a broader range of materials with electronic applications. Beginning with the 2009 conference, this third series of meetings was for Physics and Chemistry of Surfaces and Interfaces, with the sites listed in Table III.

The strength of the PCSI conference has always lay with an active program committee, whose commitment to it has

a)Electronic mail: ferry@asu.edu guaranteed the presence of invited talks at the forefront of the field. Often the set of invited talks will include both sides of a contentious issue, in order to bring to light the underlying disagreements and views that exist in the field. As mentioned, some of these themes were centerpieces of the conference for a great many years, as the scientific questions were not quickly resolved. It should be remarked that another quite popular part of the conference is to connect short presentations to each poster as well as to encourage even the invited speakers to put up a poster on their talk. This has always stimulated far more discussion for the posters as well as for the oral talks.

In the next few sections, some of these themes, for which PCSI has been an important factor, are described. A number of theoretical and experimental contributions are mentioned as they highlighted the evolution of thinking about each of the problems. But, the list is a personal choice of problems, and is not intended to be a complete discussion of the advances since the first conference.

\section{SOME DEBATE IN THE EARLY YEARS}

The first PCSI was focused mainly upon Schottky barriers and oxides on the III-V materials. It was not recognized at the time that the behavior was governed by the same defect structure, as this latter insight came later. There have been several themes that have flowed through a series of workshops. It is impossible to cover them all, but I will focus upon a few whose impact has been felt throughout the community.

\section{A. Schottky barriers and defects}

At the beginning, it was clear to all involved that the surfaces and interfaces of the compound semiconductor

TABLE I. First PCSI series.

\begin{tabular}{ll}
\hline \hline 1974 & Fort Collins, Co \\
1975 & UCLA \\
1976 & San Diego, CA \\
1977 & Princeton, NJ \\
1978 & Los Angeles, CA \\
1979 & Asilomar, CA \\
1980 & Estes Park, CO \\
1981 & Williamsburg, VA \\
\hline \hline
\end{tabular}


TABLE II Series 2 PCSI.

\begin{tabular}{ll}
\hline \hline 1982 & Asilomar, CA \\
1983 & Sante Fe, NM \\
1984 & Pinehurst, NC \\
1985 & Tempe, AZ \\
1986 & Pasadena, CA \\
1987 & Salt Lake City, UT \\
1988 & Asilomar, CA \\
1989 & Bozeman, MT \\
1990 & Clearwater Beach, FL \\
1991 & Long Beach, CA \\
1992 & Death Valley, CA \\
1993 & Williamsburg, VA \\
1994 & New Paltz, NY \\
1995 & Scottsdale, AZ \\
1996 & La Jolla, CA \\
1997 & Research Triangle, NC \\
1998 & Salt Lake City, UT \\
1999 & San Diego, CA \\
2000 & Salt Lake City, UT \\
2001 & Orlando, FL \\
2002 & Sante Fe, NM \\
2003 & Salt Lake City, UT \\
2004 & Kailua-Kona, HI \\
2005 & Bozeman, MT \\
2006 & Cocoa Beach, FL \\
2007 & Salt Lake City, UT \\
2008 & Sante Fe, NM \\
\hline & \\
\hline
\end{tabular}

materials were significantly different than those of the elemental semiconductors. Yet, the surface/interface structure itself was not clearly known for any of these materials, although there were already a number of groups addressing the problems. At the time of the first meeting, it was supposed that the presence of surface states on, e.g., GaAs was a result of specific surface atoms. ${ }^{1}$ It was felt that this was especially true for the intrinsic, or clean, surface, as much less work had been done on the over-layers such as metals, although Spicer's group was well known for this latter endeavor, and particularly for their studies in photoemission spectroscopy.

Others, however, particularly the Princeton group, performed surface studies of cleaved surfaces of these materials. They felt that the states at the surface were intrinsic surface states, perhaps arising from relaxation or reconstruction of a cleaved surface, when it was a nonpolar surface. ${ }^{2}$ This view was supported by, e.g., IBM, and clear indications of dangling bond surface states in GaAs were found. ${ }^{3,4}$

However, the situation was clouded by the claim of dominance of metal-induced gap states. These were first discussed

TABLE III. Series 3 PCSI.

\begin{tabular}{ll}
\hline \hline 2009 & \\
2010 & Santa Barbara, CA \\
2011 & Sante Fe, NM \\
2012 & San Diego, CA \\
2013 & Sante Fe, NM \\
\hline \hline
\end{tabular}

by Heine, ${ }^{5}$ but significant importance was attached to them after theoretical calculations at Berkeley. ${ }^{6}$ These latter calculations suggested that the gap states were bulk-like within the metal, and they decayed rapidly into the semiconductor. Nevertheless, they induced occupation of these states in the Schottky barrier by semiconductor carriers. Later, Tersoff ${ }^{7}$ pointed out that these were both conduction and valence like in nature, and the latter should be occupied. This suggested that the Fermi level should lie near the crossover between these two types of states. However, as we will see below, the idea of induced states was losing traction, and this latter idea went on to prominence in heterostructures. The way in which the various models fit to the wealth of experimental data has been carefully reviewed by Mönch. ${ }^{8}$

The problem of Schottky barriers was deeper than just the question of the source of the Fermi energy pinning. A part of this question had to do with whether or not the metal overlayer reacted with the semiconductor, and what the nature of this interaction could be. ${ }^{9}$ The statement that the result of the creation of the Schottky barrier was simply pinning by an intrinsic surface state or by the properties of an overlayer atom is too simplistic at first thought, as the metallurgy might well be much more complex and this could well have an important effect. Indeed, the question over whether or not there was an interfacial layer which played a crucial role in the determination of the Schottky barrier height was of considerable interest through the years. ${ }^{10,11}$ The question was heightened by Williams, who showed that the presence of an interfacial oxide or chlorine affected the electronic structure of the semiconductor, but made little effect on the magnitude of the Schottky barrier. ${ }^{12,13} \mathrm{He}$ also questioned the $S$ parameter that had been earlier suggested as a characteristic of Schottky barriers. ${ }^{14}$

Almost separately, other groups worried about the nature of the oxides that were put on the compound semiconductors. ${ }^{3}$ It was known that trying to make a MOS structure on GaAs was quite difficult, and these devices just did not seem to have a future. But, putting an oxide on InP gave a situation with a much lower density of surface states. ${ }^{15}$ But, at the beginning, it was not appreciated that these studies might well be connected to those of the Schottky barrier.

Nevertheless, as time went by, the views of these distinct surfaces began to merge quite rapidly. By the time of Asilomar 2 (1982), the commonality was established, and it was now believed that Fermi level pinning did not depend upon what was put on the surface, but it did depend upon what the cation was. Thus, Ga compounds seem to be doomed to a pinned Fermi level, but In compounds had a much lower density of defect levels and could be used for MOS transistors.

\section{B. Heterojunction band alignments}

The question of the alignment of the bands of two distinct semiconductors when they were joined to form a heterostructure also was a key issue at the first PCSI. As Kroemer expressed it: "All of these devices depend to a varying degree on the details of the band structure of the 
heterojunctions, especially on the way in which the total energy gap discontinuity

$$
\Delta \varepsilon_{G}=\varepsilon_{G 2}-\varepsilon_{G 1}=\Delta \varepsilon_{C}+\Delta \varepsilon_{V}
$$

of an (abrupt) heterojunction is divided into a conduction band discontinuity $\Delta \varepsilon_{C}$ and a valence band discontinuity $\Delta \varepsilon_{V}, \ldots .$, This became a central issue which propagated through the conference for a great many years. To begin, Kroemer considered the so-called electron-affinity rule and found it very unsatisfying. In short, this rule aligns the bands of the two materials such that the conduction bands each lie the proper distance from the vacuum level that corresponds to their electron affinity. One problem pointed out is that this quantity is a surface quantity and not really relevant to a buried interface.

At the second conference, Ray Dingle presented the results of optical studies of an array of heterojunction quantum wells. ${ }^{17}$ By studying the electron-hole transitions, they were able to infer that $88 \%$ of the band discontinuity lay in the conduction band. While the measurements were very nice, the number which they inferred came under attack almost immediately, with a smaller value ultimately being determined. But, it was also determined that the use of the square wells did not allow a unique determination of the band offset. ${ }^{18}$ The problem was that as one moved the band offset from the conduction band to the valence band, the electron quantum well levels would move to lower energies, and at the same time, the hole levels would also move, in the same absolute direction. Since these levels moved like $\sim 1 / L^{2}$, it was exceedingly difficult to pin down the proper band offset. In the end, the best estimate came from single band measurements utilizing internal photoemission. ${ }^{19}$ These measurements suggested that the conduction band offset was closer to $62 \%$ of the band offset, a number that is still considered to be believable today.

But, the question remained: "How does one predict the band offset in the heterostructures?" Or, if the electron affinity rule was not going to work, what would? This has led to a long history of suggestions. One of the first was the common-anion rule. ${ }^{20}$ It was noted that in semi-empirical tightbinding calculations of the semiconductor band structure, ${ }^{21}$ the valence band maximum was invariably given by the anion $p$-state wave functions. It was then obvious that, for a material like GaAlAs/GaAs, there should be no discontinuity in the valence band, and nearly all of the discontinuity should be in the conduction band, in keeping with the earlier optical measurements. But, this approach was not very successful for GaAlAs/GaAs, much less for many other heterostructures.

Kroemer set out to see if bulk band structures could be used to predict the discontinuity in the heterostructure bands. $^{22}$ The problem lay in trying to predict just which point in the Brillouin zone should be used to try to create the cross interface boundary. It was suggested that an average potential might be the route forward. But, what the bulk calculations missed is the inevitable interface dipole. That is, there is a break in the local bonding structure as one crosses the interface, and charge penetration from one side to the other leads to an interface dipole. ${ }^{23}$ Such dipoles lead to potential shifts between the two sides of the interface. One needed to be able to determine this dipole in order to predict the band lineup of the heterostructure. However, the concept of the total energy bands suggested to many that by measuring the core levels of the various constituent atoms, one could estimate the band shifts, and therefore determine the band offsets.

By 1981, results of experiments on measuring the core level shifts across the heterointerface were beginning to appear, and the results were not pretty. To be sure, it is a difficult experiment since photoemission requires the generated electrons to be close enough to the surface to be emitted and measured. Thus, the heterointerface needs to be close to the surface and therefore requires quite thin epitaxial layers. With this in mind, the results began to arrive. As an example, one study showed that growth of AlAs on top of GaAs led to a valence band discontinuity (average) of $0.4 \mathrm{eV}$, but growth of a GaAs layer on top of AlAs gave a valence band discontinuity of only $0.15 \mathrm{eV} .{ }^{24}$ Now, it was known to be possible to grow atomically sharp interfaces when AlAs was grown on GaAs, but the reverse order produced rougher interfaces. It was obvious that this led to a different interfacial dipole. Finding the "right" band offsets was not going to be easy, and the results were likely going to depend upon how the material was grown.

It was also suggested that one could use the presence of deep levels to infer how the bands should be aligned in the heterostructure. It was proposed that, in cases where the interface was atomically abrupt, bulk properties might well be usable to predict the band lineups, and that an appropriate bulk level would arise from the transition-metal impurities. ${ }^{25}$ These impurities are known to form deep levels within most semiconductors. But, since these levels do not seem to be associated with either the conduction band or the valence band, they should provide a good reference level for alignment of the bands in heterostructures. This argument predicts a valence band offset in AlAs/GaAs of about $0.34 \mathrm{eV}$, which is close to the 0.35 predicted by Frensley and Kroemer, ${ }^{26}$ and not far from that found from the photoemission above, even though this predicted number ignores their calculated charge transfer dipole.

In subsequent calculations, it was pointed out that a dangling bond level associated with the transition-metal atoms played the role of the "neutrality" level, and led to the correlation found from using these atoms to predict band lineups. $^{27}$ Earlier, it was noted that the average hybrid energy, determined from tight-binding calculations, played a role not unlike the neutrality level discussed above for Schottky barriers. $^{28}$ Indeed, the connection between Schottky barriers and the band lineup for various oxides on Si was again discussed by Robertson in connection with the advent of high- $\kappa$ dielectrics. $^{29}$

In some sense, a closure was arising, but it always seemed like good agreement was obtained after the measurements were made, and that the theories were not sufficiently predictive in nature. Perhaps, the status was (and is) best described 
by a statement from Charley Duke. I believe it was at the first Sante Fe conference (1983), when Duke was chairing a theory session. A young Asian scientist ran into some difficulty with questions. At this point, the chair cut off debate by stating what I now call Duke's Law:

"All theory is wrong; it is just a matter of degree."

Needless to say, there is always a corollary, in this case coming from Wolf Porod:

"All experiment is correct; it is just a matter of what is measured."

\section{Contacts}

Contacts on III-V materials have always been a problem, and there has been interest at PCSI since the beginning. In $\mathrm{Si}$, for example, "good" electrical contacts are made by doping the material beneath the contact metal to a very high level. This makes an extremely thin Schottky depletion layer, and electrons readily tunnel through this layer. But, most of the III-V materials cannot be doped to such high levels, and therein lies the problem. Putting the metal down just creates a Schottky barrier which impedes the motion of carriers to the semiconductor. In fact, many felt that this problem would not be solved until the Schottky barrier was fully understood.

Secondarily, there was a problem with the fact that the actual tunneling coefficient that was calculated did not agree well with any measurements (usually too low a value of calculated tunneling). At first, it was suggested that one needed to use a more sophisticated multiband model with a dynamic dielectric function and properly account for image charge and trapped negative charge. ${ }^{30}$ But, it was shortly realized that, with the small effective mass in these materials, one had to account for the discrete nature of the doping, and use the exact positions of the impurities in the solving of Poisson's equation. ${ }^{31}$ The random nature of the dopant caused some impurities to be closer to the metal, and this then led to as much as half an order of magnitude increase in tunneling. This was a harbinger of important effects soon to be crucial to the device industry.

Keyes had first suggested that the small number of dopant atoms in small transistors would lead to fluctuations in current that could not be ignored. ${ }^{32}$ The statistical fluctuations that would arise from a small number of impurities would dramatically affect the device performance. The above variation in contact resistance was a signal of the arrival of this problem. ${ }^{31}$ Then, Joshi considered the random impurities in bulk GaAs and showed that each electron was, on average, interacting with three impurities at any one time, ${ }^{33}$ which heralded the importance of multiple-scattering. Soon afterward, Wong and Taur presented the results of this random impurity distribution on MOSFETs ${ }^{34}$ clearly demonstrating that it was important in future generation of devices. Our own group demonstrated the importance in III-V devices. ${ }^{35}$ The importance of interface roughness at the oxidesemiconductor interface had earlier been emphasized. ${ }^{36}$ Now, line-edge roughness, ${ }^{37}$ which led to fluctuations in channel length, and even interface roughness between the gate metal and the oxide were shown to produce important effects in devices. ${ }^{38}$ It was clear that fluctuations arising from randomness in the device structure were going to be an important problem within the short term future.

Relief of the contact problem came with the introduction of the alloyed AuGeNi contact formulation. It was discovered that a number of intermetallic compounds were formed, if the alloying was done at the proper temperature. ${ }^{39,40}$ In the intervening years, many other intermetallic compounds have been used to create acceptable contacts. ${ }^{41}$

Until this point, I have not addressed the fact that most people do not consider contacts to be important aspects of their research. This is a mistake, as contacts may be the most critical part of an experimental structure. Bad aspects can include injection of carriers and current limiting behavior, just to name two contrary effects. We generally hear about the problem of injection of carriers, but most are not familiar with current limiting behavior. ${ }^{42}$ But, a simple example illustrates this very efficiently. Over the past decade or so, there has been interest in transport in $\mathrm{GaN}$, and its heterostructure with $\mathrm{AlGaN}$. Yet, measurements of the velocity-field curve gave current/velocity values much below those expected from theory. It was only when the titanium rich Ti:Au metallurgy was developed that these ohmic contacts allowed sufficient current for the samples to demonstrate their very high velocities. ${ }^{43,44}$ Thus, the understanding of the role that contacts play in the experiment is crucial as one addresses new materials, especially for electronic applications.

\section{FAST FORWARD}

Through the years, PCSI has had a history of creating focus sessions, in which a new or evolving "effect" is brought to the attention of the attendees. Quite often, this arises through a special session, with multiple papers so that various sides of the scientific debate can be brought together to give a fuller view of the issues. Among the topics in recent years has been graphene, two-dimensional electron (or hole) gasses at the interface of two normal insulating oxides, and recently topological insulators. I will focus here only upon the last two of these topics, because there are some practical issues involved in these particular fields.

\section{A. Oxide interfaces}

In normal HEMTs, the two-dimensional electron gas is derived from dopants, usually placed within the "insulating" layer. That is, in GaAs HEMTs, the dopants are placed in the AlGaAs layer via what is known as modulation doping. ${ }^{45}$ This approach is used in nearly all III-V based HEMTs. However, wurtzite materials such as $\mathrm{GaN}$ and $\mathrm{AlGaN}$ possess a spontaneous polarization. Naturally, the amplitudes of the polarization are different in these two materials, and an interface will see the discontinuity in this polarization. As a result, a two-dimensional electron (or hole) gas will form at the interface to provide the charge required to balance this polarization discontinuity. ${ }^{46}$

But, polar imbalance has been discovered in nonsemiconductor materials. Recently, two oxide insulators have revealed 
the existence of a quasi-two-dimensional electron gas at the interface between them, when one is grown on the other by molecular-beam epitaxy. ${ }^{47}$ In this case, both oxides are wide bandgap insulating perovskites, yet they produce this semiconductor behavior when the heterointerface is formed. $\mathrm{SrTiO}_{3}$ is a typical $\mathrm{ABO}_{3}$ material, in which the structure can be considered as alternate layers of $\mathrm{BO}_{2}$ and $\mathrm{AO}$. Both of these layers are charge neutral, so there is nothing immediately weird at this point. However, the similar $\mathrm{LaAlO}_{3}$ has sheets that are charged by $\pm e / 2$, and this changes the net behavior. The growth by molecular-beam epitaxy allows one to form the structure basically layer by layer. When the interface was $\left(\mathrm{AlO}_{2}\right)^{-}(\mathrm{SrO})^{0}$, a hole layer was expected, but the interface remained insulating. On the other hand, when the interface was $(\mathrm{LaO})^{+}\left(\mathrm{TiO}_{2}\right)^{0}$, an electron gas was pulled into the interface. The electrons exhibited a very high mobility and quantum behavior at low temperatures.

In this latter situation, as in the HEMTs above and MOSFETs, the electrons in the interfacial inversion layer are drawn into the structure from the contacts, if there is no intrinsic source. Presumably, there are traps which absorb the holes in the first situation. The presence of the electrons at the interface is symbolic of the fact that nature prefers charge neutrality. Even in transistor structures, where space regions are engineered, global charge neutrality is maintained. If this did not occur, it would be shocking indeed.

The above interpretation is nice, but there are many problems existing before a full understanding can be obtained. Not the least of the problems, is the fact that Hall measurements on the above structure leads one to believe that there is an incredibly large electron density in the inversion layer. ${ }^{47}$ And, there is the role of strain in these layers. ${ }^{48}$ It appears that the carriers have strong correlation, which can lead to superconductivity ${ }^{49}$ and magnetism, ${ }^{50}$ which, unusually, can coexist. ${ }^{51}$ This is even before we consider that other oxides can be used for these effects. It is clear that this is a new and exciting field, which may well have an important impact in electronics.

\section{B. Topological insulators}

Topological insulators have been all the rage the past few years, so it is natural that they have come to PCSI. A reasonably recent review of the subject can be found in Ref. 52. Of key interest for electronic properties is that these materials often possess a Dirac-like band structure, which is topologically stabilized at a surface or interface. One prototypical material is a heterostructure between the negative band gap material $\mathrm{HgTe}$ and the positive band gap HgCdTe or CdTe. In HgTe, the energy state that composes the normal top of the valence band and the one that normally is the bottom of the conduction band are inverted. When crossing the interface between the two materials, this band ordering must reverse itself, and this can lead to the Dirac-like band structure at the interface, especially with the large spin-orbit interaction in $\mathrm{HgTe}$. For the discussion here, it is not the presence of a topological state in this heterostructure, but the question as to why this property was not discovered much earlier.
The nature of bands in the $\mathrm{HgTe} / \mathrm{CdTe}$ superlattice was being discussed at PCSI at least as early as $1979 .{ }^{53,54}$ So, the nature of the energy structure in the interface between the two materials must have been known, especially as the spin-orbit interaction was specifically included. But, there may well have been two reasons that the interesting interfacial properties were not appreciated. First, the concepts of topology were not in the mainstream of condensed matter and materials physics at the time. Second, the intriguing ideas of Dirac-like bands in semiconductors were not appreciated at the time.

In one sense, topology crept into the field with Laughlin's introduction of a structure and phase rigidity to explain the accuracy of the quantum Hall effect. ${ }^{55}$ It became stronger with the discussion of fractionally charged quasiparticles in the fractional quantum Hall effect. In the latter, the filling factor for various Hall states is the ratio of the number of electrons to the number of flux quanta in the structure. Thus, in the $\nu=1 / 3$ state, there are three flux quantum for each electron. When the magnetic field is exactly at this filling factor, all the states in this level are completely full. If we now add a single flux quantum, then the unoccupied state must hold a quasihole with a charge of $1 / 3$ to fit the statistics, ${ }^{56,57}$ a fact that has been verified with experiment. ${ }^{58}$ It is generally believed that these statistics are best described by a Chern-Simons gauge theory, ${ }^{59}$ and this leads to a connection to quantum computing. ${ }^{60}$ The interaction between two fractional states has shown the phase interference that would be necessary for this purpose. ${ }^{61}$ So, topology and topological computing came to the forefront with these fractional charge states, and the genie was out of the bottle.

Appreciation of the Dirac-like band structure came later. The details of this band structure have been known for decades. ${ }^{62}$ But, it was only with the isolation of single layers of graphene $^{63}$ that experiments could begin to establish the unique properties of this band structure. And, studies of the quantum Hall effect quickly illustrated that these bands had different properties than normal semiconductors. ${ }^{64}$

So, by 2005 or so, there was a reasonable appreciation of the role of topology in condensed matter physics, and there was demonstrated different behavior in Dirac-like bands. At this point, the stage was set for someone to suggest a more general view of topological insulators (although the idea of a topological superconductor is older ${ }^{65}$ ), and the ideas on topological changes were widespread and growing a few years earlier. For example, the topological transition between a correlated insulator and a band insulator was discussed about this time. ${ }^{66}$ In spite of this nice argument, the phrase "topological insulator" actually seems to have originated in biology, ${ }^{67}$ but burst into condensed matter theory at about the same time. ${ }^{68,69}$ Today, the focus is more directed at materials (and structures) which have insulating bulk properties, but exhibit metallic surfaces. ${ }^{52}$ One hopes that we will continue to hear more about these at future PCSI conferences.

\section{SUMMARY}

PCSI has continued to be an important, and interesting, conference over the past 40 years. This is best exemplified 
by the continued strong presence. Perhaps more importantly, the conference continues to draw a sizable number of young (student) attendees, which points to the strong environment for discussion and interaction which the conference provides. Thus, it appears to have the necessary requisites to continue for another 40 years.

\section{ACKNOWLEDGMENTS}

The author would like to especially thank Rudy Ludeke for his aide in recalling the early years of PCSI, particularly with respect to the publications and some important people. However, the neglect of some important contributions is entirely my own failure.

${ }^{1}$ W. E. Spicer and P. E. Gregory, CRC CR Solid State Sci. 5, 231 (1975).

${ }^{2}$ P. Mark, S. C. Chang, W. F. Creighton, and B. W. Lee, CRC CR Solid State Sci. 5, 189 (1975).

${ }^{3}$ R. Ludeke and A. Koma, CRC CR Solid State Sci. 5, 259 (1975).

${ }^{4}$ J. L. Freeouf and D. E. Eastman, CRC CR Solid State Sci. 5, 245 (1975).

${ }^{5}$ V. Heine, Phys. Rev. 138, A1689 (1965).

${ }^{6}$ S. G. Louie, J. R. Chelikowsky, and M. L. Cohen, J. Vac. Sci. Technol. 13, 790 (1976).

${ }^{7}$ J. Tersoff, Phys. Rev. Lett. 52, 465 (1984).

${ }^{8}$ W. Mönch, in GaAs Technology II, edited by D. K. Ferry (Sams, Carmel, IN, 1990), pp. 139-172.

${ }^{9}$ G. Y. Robinson and D. J. Fertig, CRC CR Solid State Sci. 5, 291 (1975).

${ }^{10}$ W. J. Schaffer, R. W. Bené, and R. M. Walser, J. Vac. Sci. Technol. 15, 1325 (1978).

${ }^{11}$ L. J. Brillson, J. Vac. Sci. Technol. 16, 1137 (1979).

${ }^{12}$ R. H. Williams, R. R. Varma, and V. Montgomery, J. Vac. Sci. Technol. 16, 1418 (1979).

${ }^{13}$ T. P. Humphreys, M. H. Patterson, and R. H. Williams, J. Vac. Sci. Technol. 17, 886 (1980).

${ }^{14}$ S. Kurtin, T. C. McGill, and C. A. Mead, Phys. Rev. Lett. 22, 1433 (1969).

${ }^{15}$ C. W. Wilmsen, CRC CR Solid State Sci. 5, 313 (1975).

${ }^{16}$ H. Kroemer, CRC CR Solid State Sci. 5, 555 (1975).

${ }^{17}$ R. Dingle, W. Wiegmann, and C. H. Henry, Phys. Rev. Lett. 33, 827 (1974).

${ }^{18}$ W. Porod, W. Pötz, and D. K. Ferry, J. Vac. Sci. Technol. B 3, 1290 (1985).

${ }^{19}$ M. Heiblum, M. I. Nathan, and M. Eizenberg, Appl. Phys. Lett. 47, 503 (1985).

${ }^{20}$ J. O. McCaldin, T. C. McGill, and C. A. Mead, Phys. Rev. Lett. 36, 56 (1976).

${ }^{21}$ J. C. Slater and G. F. Koster, Phys. Rev. 94, 1498 (1954).

${ }^{22}$ W. R. Frensley and H. Kroemer, J. Vac. Sci. Technol. 13, 810 (1976).

${ }^{23}$ W. R. Frensley and H. Kroemer, Appl. Phys. Lett. 31, 48 (1977).

${ }^{24}$ J. R. Waldrop, S. P. Kowalczyk, R. W. Grant, E. A. Kraut, and D. L. Miller, J. Vac. Sci. Technol. 19, 573 (1981).

${ }^{25}$ J. M. Langer and H. Heinrich, Phys. Rev. Lett. 55, 1414 (1985).

${ }^{26}$ W. R. Frensley and H. Kroemer, Phys. Rev. B 16, 2642 (1977).

${ }^{27}$ J. Tersoff and W. A. Harrison, Phys. Rev. Lett. 58, 2367 (1987).

${ }^{28}$ W. A. Harrison and J. Tersoff, J. Vac. Sci. Technol. B 4, 1068 (1986).
${ }^{29}$ J. Robertson, J. Vac. Sci. Technol. B 18, 1785 (2000).

${ }^{30}$ W. J. Boudville and T. C. McGill, J. Vac. Sci. Technol. B 3, 1192 (1985).

${ }^{31}$ W. J. Boudville and T. C. McGill, Appl. Phys. Lett. 48, 791 (1986).

${ }^{32}$ R. W. Keyes, Science 195, 1230 (1977).

${ }^{33}$ R. P. Joshi and D. K. Ferry, Phys. Rev. B 43, 9734 (1991).

${ }^{34}$ H.-S. Wong and Y. Taur, IEDM Conf. Proc. (IEEE, New York, 1993), p. 705.

${ }^{35}$ J.-R. Zhou and D. K. Ferry, IEEE Comp. Sci. Eng. 2, 30 (1995).

${ }^{36}$ S. M. Goodnick, D. K. Ferry, C. W. Wilmsen, Z. Lilienthal, D. Fathy, and O. L. Krivanek, Phys. Rev. B 32, 8171 (1985).

${ }^{37}$ T. D. Linton, S. Yu, and R. Shaheed, VLSI Des. 13, 103 (2001).

${ }^{38}$ M. J. Rack, D. Vasileska, D. K. Ferry, and M. Sidorov, J. Vac. Sci. Technol. B 16, 2165 (1998).

${ }^{39}$ M. Heiblum, M. I. Nathan, and C. A. Chang, Solid State Electron. 25, 185 (1982).

${ }^{40}$ T. S. Kuan, P. E. Batson, T. N. Jackson, H. Rupprecht, and E. L. Wilkie, J. Appl. Phys. 54, 6952 (1983).

${ }^{41}$ A. G. Baca, F. Ren, J. C. Zolper, R. D. Briggs, and S. J. Pearton, Thin Solid Films 308-309, 599 (1997).

${ }^{42}$ H. Kroemer, IEEE T. Electron Dev. 15, 819 (1968).

${ }^{43}$ J. M. Barker, D. K. Ferry, S. M. Goodnick, D. D. Koleske, A. Allerman, and R. Shul, J. Vac. Sci. Technol. B 22, 2045 (2004).

${ }^{44}$ J. M. Barker, D. K. Ferry, D. D. Koleske, and R. Shul, J. Appl. Phys. 97, 063705 (2005).

${ }^{45}$ R. Dingle, H. L. Störmer, A. C. Goddard, and W. Wiegman, Appl. Phys. Lett. 33, 665 (1978).

${ }^{46}$ O. Ambacher et al., J. Appl. Phys. 85, 3222 (1999).

${ }^{47}$ A. Ohtomo and H. Y. Hwang, Nature 427, 423 (2004).

${ }^{48}$ C. W. Bark et al., Proc. Natl. Acad. Sci. U.S.A. 108, 4720 (2011).

${ }^{49}$ N. Reyren et al., Science 317, 1196 (2007).

${ }^{50}$ A. Brinkman et al., Nat. Mater. 6, 493 (2007).

${ }^{51}$ L. Li, C. Richter, J. Mannhart, and R. C. Ashoori, Nat. Phys. 7, 762 (2011).

${ }^{52}$ J. Moore, IEEE Spectr. 48, 38 (2011).

${ }^{53}$ J. N. Schulman and T. C. McGill, J. Vac. Sci. Technol. 16, 1513 (1979).

${ }^{54}$ J. N. Schulman and T. C. McGill, J. Vac. Sci. Technol. 17, 1118 (1980).

${ }^{55}$ R. B. Laughlin, Surf. Sci. 113, 22 (1982).

${ }^{56}$ F. Wilczek, Phys. Rev. Lett. 48, 1144 (1982); 49, 957 (1982).

${ }^{57}$ B. Halperin, Helv. Phys. Acta 56, 75 (1983).

${ }^{58}$ V. J. Goldman and B. Su, Science 267, 1010 (1995).

${ }^{59}$ C. Nayak and F. Wilczek, Nucl. Phys. B 479, 529 (1996).

${ }^{60}$ A. Stern, F. von Oppen, and E. Mariani, Phys. Rev. B 70, 205338 (2004).

${ }^{61}$ F. E. Camino, W. Zhou, and V. J. Goldman, Phys. Rev. B 72, 075342 (2005).

${ }^{62}$ P. R. Wallace, Phys. Rev. 71, 622 (1947).

${ }^{63}$ K. S. Novoselov, A. K. Geim, S. V. Morozov, D. Jiang, Y. Zhang, S. V. Dubonos, I. V. Grigorieva, and A. A. Firsov, Science 306, 666 (2004).

${ }^{64}$ K. S. Novoselov, A. K. Geim, S. V. Morozov, D. Jiang, M. I. Katsnelson, I. V. Grigorieva, S. V. Dubonos, and A. A. Firsov, Nature 438, 197 (2005).

${ }^{65}$ P. Wiegmann, Prog. Theor. Phys. 107, 243 (1992).

${ }^{66}$ A. A. Aligia, K. Hallberg, B. Normand, and A. P. Kampf, Phys. Rev. Lett. 93, 076801 (2004).

${ }^{67}$ L. Moulin, A. R. Rahmouni, and F. Boccard, Mol. Microbiol. 55, 601 (2005).

${ }^{68}$ Y. Hatsugai, J. Phys. Soc. Jpn. 74, 1374 (2005).

${ }^{69}$ M. B. Hastings, Europhys. Lett. 70, 824 (2005). 